\title{
An All-Interaction Matrix Approach to Linear and Bilinear System Identification
}

\author{
Minh Q. Phan, Francesco Vicario, Richard W. Longman, and Raimondo Betti
}

\begin{abstract}
This paper is a brief introduction to the interaction matrices. Originally formulated as a parameter compression mechanism, the interaction matrices offer a unifying framework to treat a wide range of problems in system identification and control. We retrace the origin of the interaction matrices, and describe their applications in selected problems in system identification.
\end{abstract}

\section{Introduction}

The interaction matrices were originally motivated by the system identification of large flexible space structures [1]. Because such structures are lightly damped, a very large number of system Markov parameters need to be solved for. The interaction matrix was first formulated by the first author as a mechanism to compress an infinite sequence of system Markov parameters into a finite sequence which can be identified more easily [2,3]. The system Markov parameters are then recovered from the compressed Markov parameters, and the recovery can be achieved without having to know the interaction matrix that performs the compression in the

Minh Q. Phan

Thayer School of Engineering, Darmouth College, Hanover, NH 03755, USA e-mail: mqphan@dartmouth.edu

Francesco Vicario

Philips Research North America, Briarcliff Manor, NY 10510, USA e-mail: francesco.vicario@philips.com (at Columbia University when this work was done)

Richard W. Longman

Department of Mechanical Engineering, Columbia University, New York, NY 10027, USA e-mail: rw14@columbia.edu

Raimondo Betti

Department of Civil Engineering and Engineering Mechanics, Columbia University, New York, NY 10027, USA e-mail: betti@ civil.columbia.edu 
first place. Since this original development, utilities of the interaction matrices in a wide range of applications have been discovered due to their versatility in connecting the state-space model to various input-output output representations. This paper explains some of our own work in this area. Applications of the interaction matrices have been found in Observer/Kalman filter identification (OKID) [4]-[10] including its output-only version $\left(\mathrm{O}^{3} \mathrm{KID}\right)$ [11], closed-loop system identification [12],[13], modal discrimination [14], multi-step ahead state estimation [15],[16], system identification in the presence of periodic disturbances [17]-[20], structural damage monitoring [21], actuator failure detection [22], data-based, model-based, and decentralized model predictive control [23],[24], tracking control of unmeasured outputs [25], control of vehicle formations [26],[27], iterative learning control [28], linear time-varying system identification [29],[30], identification of discretetime bilinear systems by equivalent linear models [31], [32], nonlinear ARX models [33], superspace methods [34], [35], subspace intersection and subspace projection methods via switched linear systems [36]-[39], and optimal bilinear observers and bilinear OKID [40]-[42] in the presence of noisy measurements. This paper explains the interaction matrices in selected system identification problems. The role of the interaction matrices in the control problems is explained in more details in [43].

\section{The System Markov Parameters in Context}

The system Markov parameters naturally arose in the problem of identifying a linear state-space system of the form:

$$
\begin{aligned}
x(k+1) & =A x(k)+B u(k) \\
y(k) & =C x(k)+D u(k)
\end{aligned}
$$

Given a set of sufficiently rich and long input-output data record denoted by $\{u(k)\}$ and $\{y(k)\}$ starting from some unknown initial state $x(0)$, we seek a realization of the original $A, B, C, D$ model whose input-output relationship is

$$
y(k)=C A^{k} x(0)+D u(k)+\sum_{i=1}^{k} C A^{i-1} B u(k-i)
$$

The combinations $D, C B, C A B, C A^{2} B, \ldots$ are called the system Markov parameters. If a sufficient number of systems Markov parameters is known, they can be factored to find a realization of $A, B, C$. Although the factorization problem is non-linear, it has an exact analytical solution. Because the Markov parameters are linearly related to input-output data, it might appear that their direct identification is trivial, but this initial impression is false. Writing Eq. (2) for $k=0,1,2, \ldots$ produces

$$
\begin{aligned}
& y(0)=C x(0)+D u(0) \\
& y(1)=C A x(0)+D u(1)+C B u(0) \\
& y(2)=C A^{2} x(0)+D u(2)+C B u(1)+C A B u(0), \ldots
\end{aligned}
$$


The number of equations to be written is a function of the number of system Markov parameters that one wishes to solve for. Treating the scalar elements of the combinations $C x(0), C A x(0), C A^{2} x(0)$ and $D, C B, C A B$ as unknowns, simple counting reveals that the number of unknowns exceeds the number of linear equations. Thus the Markov parameters cannot be solved from these linear equations alone. The additional equations which describe the relationship among the Markov parameters are non-linear $(k \geq 0)$,

$$
C A^{k+n} B+a_{n-1} C A^{k+n-1} B+\cdots+a_{1} C A^{k+1} B+a_{0} C A^{k} B=0
$$

because they involve the product of the Markov parameters and the $n$ unknown coefficients of the characteristic equation of $A$ (where $n$ itself is unknown),

$$
\lambda^{n}+a_{n-1} \lambda^{n-1}+\cdots+a_{1} \lambda+a_{0}=0
$$

Thus the mathematical problem of finding the Markov parameters directly from input-output data is surprisingly more challenging than one would expect. Other approaches would solve for the Markov parameters indirectly via an infinite impulse response (IIR) model (an exact method), or the Fast Fourier Transform (FFT) technique (an approximate method).

\section{Origin of the Interaction Matrix}

The interaction matrix offers another approach to solve for the Markov parameters that is both intuitively and mathematically attractive. A matrix $M$, referred to as an interaction matrix $[2,3]$, was introduced as a mechanism to compress an infinite sequence of Markov parameters of the original system,

$$
\left\{D, C B, C A B, C A^{2} B, \ldots\right\}
$$

to a finite sequence,

$$
\left\{D, C \bar{B}, C \bar{A} \bar{B}, \ldots, C \bar{A}^{p-1} \bar{B}\right\}
$$

where $\bar{A}$ and $\bar{B}$ are related to the original $A$ and $B$ via $M$,

$$
\bar{A}=A+M C \quad \bar{B}=\left[\begin{array}{ll}
B+M D & -M
\end{array}\right]
$$

The parameter $p$, which controls the number of compressed Markov parameters, must satisfy the condition $p m \geq n$, where $m$ is the number of independent outputs and $n$ the dimension of the state of the system being identified. The compression mechanism by the interaction matrix $M$ limits the number of unknowns. Once the compressed Markov parameters are identified from input-output data, they can be uncompressed to recover any number of original Markov parameters. Moreover, the uncompression can be done without having to find the interaction matrix $M$ that provides the compression in the first place. To see how the interaction matrix is 
introduced to (1), add and subtract $M y(k)$ to the state equation,

$$
\begin{aligned}
x(k+1) & =A x(k)+B u(k)+M y(k)-M y(k) \\
& =(A+M C) x(k)+(B+M D) u(k)-M y(k)
\end{aligned}
$$

Define a new "input" vector $v(k)$ as $v(k)=\left[\begin{array}{ll}u(k) & y(k)\end{array}\right]^{T}$ the original state-space model (1) becomes

$$
\begin{aligned}
x(k+1) & =\bar{A} x(k)+\bar{B} v(k) \\
y(k) & =C x(k)+D u(k)
\end{aligned}
$$

The input-output map of the modified state-space model can be expressed in terms of their own Markov parameters as

$$
y(k)=C \bar{A}^{k} x(0)+D u(k)+\sum_{i=1}^{k} C \bar{A}^{i-1} \bar{B} v(k-i)
$$

The interaction matrix $M$ alters the dynamics of the original system from $A$ to $\bar{A}=A+M C$. The freedom introduced by $M$ is now used to impose the condition $(A+M C)^{p}=0$ so that, for $k \geq p$, the input-output map becomes

$$
y(k)=D u(k)+\sum_{i=1}^{p} C \bar{A}^{i-1} \bar{B} v(k-i)
$$

To produce (10) from (9) the interaction matrix $M$ eliminates the explicit dependence on the unknown initial condition $x(0)$, and at the same time causes the number of Markov parameters of the modified system to become finite because $C \bar{A}^{i-1} \bar{B}=0$ for $i \geq p+1$. We are not solving for $M$ given $A$ and $C$ to satisfy $(A+M C)^{p}=0$ because both $A$ and $C$ are unknown. We are only concerned with the existence of $M$ such that $(A+M C)^{p}=0$. For an observable system, the existence of $M$ is guaranteed, e.g., one that would place all eigenvalues of $A+M C$ at the origin.

The Markov parameters of the original system can be recovered from the compressed Markov parameters without the need to find $M$. Each compressed system Markov parameter $C \bar{A}^{i-1} \bar{B}, i=1,2, \ldots, p$, has two parts, e.g., $[C(B+M D),-C M]$, $[C(A+M C)(B+M D),-C(A+M C) M]$, etc. By working these two parts against each other (together with $D$ which can be directly identified), it is possible to recover any number of system Markov parameters without actually knowing $M$. For example, the first two system Markov parameters can be solved as follows,

$$
\begin{gathered}
C B=C(B+M D)-(C M) D \\
C A B=C(A+M C)(B+M D)-C(A+M C) M D-(C M)(C B)
\end{gathered}
$$

Any number of system Markov parameters beyond $C A^{p-1} B$ can be recovered by using the condition $C \bar{A}^{i} \bar{B}=0$ for $i \geq p$. 


\section{Relationship to ARX and ARMAX Models}

The interaction matrix reveals a very useful connection between the state-space model and well-known input-output models such as ARX (Auto-Regressive with eXogenous input) and ARMAX (Auto-Regressive Moving Average with eXogenous input) models. An ARX model has the form

$$
y(k)=\sum_{i=1}^{p} \alpha_{i} y(k-i)+\sum_{i=0}^{p} \beta_{i} u(k-i)
$$

The coefficients of the ARX model are related to the state-space model matrices according to $\alpha_{i}=-C(A+M C)^{i-1} M, \beta_{i}=C(A+M C)^{i-1}(B+M D), \beta_{0}=D$. In the presence of process and measurement noises (Gaussian, white, zero-mean, uncorrelated) with unknown covariances, it can be shown that when $p$ is sufficiently large such that $(A+K C)^{p} \approx 0$, the ARX model coefficients, identified by simple least-squares from a sufficiently long input-output data record, subsumes a Kalman filter with gain $K$, and the ARX model residual converges to the Kalman filter residual. The interaction matrix $M$ in this case plays the role of the steady-state Kalman filter gain $K$. The above relationship is the theoretical basis for the original Observer/Kalman filter Identification (OKID) algorithm which finds a statespace model and an optimal observer/Kalman filter gain from noise-corrupted inputoutput data alone without knowing the process and output noise covariances [4]

In the case of an ARMAX model,

$$
y(k)=\sum_{i=1}^{p} \bar{\alpha}_{i} y(k-i)+\sum_{i=0}^{p} \bar{\beta}_{i} u(k-i)+\sum_{i=1}^{p} \bar{\gamma}_{i} \varepsilon(k-i)+\varepsilon(k)
$$

it can be shown that the ARMAX model coefficients are related to the original statespace model matrices through both $M$ and $K$ according to $\bar{\alpha}_{i}=-C(A+M C)^{i-1}$, $\beta_{0}=D, \bar{\beta}_{i}=C(A+M C)^{i-1}(B+M D), \bar{\gamma}_{i}=C(A+M C)^{i-1}(M-K)$. The order $p$ of the ARMAX model needs not be the same as the order $p$ of the ARX model, $M$ is a deadbeat observer gain, and $K$ is the steady-state Kalman filter gain. Understanding this structural connection is useful because it allows for the recovery of a state-space model $A, B, C, D$, and $K$ (and $M$ ) from the identified coefficients of an ARMAX model of "small" order. This is the basis of the OKID algorithm with residual whitening [6]. Observe that if the order $p$ of the ARMAX model is sufficiently large, then there exists an interaction matrix $M$ that approaches the Kalman filter gain $K,(A+M C)^{p} \approx 0$. When $M=K$, all the $\bar{\gamma}_{i}$ coefficients vanish, and the ARMAX model (14) reverts back to the ARX model (13). A recent extension of OKID uses the Kalman residual, which can be computed without having to find the Kalman filter first, to convert a stochastic system identification problem into a deterministic problem. Any deterministic state-space identification algorithms, including subspace and non-subspace algorithms, can then be used to find a state-space model of the system and a corresponding Kalman filter gain [10]. 


\section{A Generalized Interaction Matrix}

The adding and subtracting of $M y(k)$ in (7) leads to a condition on the interaction matrix $M$ that is nonlinear, $(A+M C)^{p}=0$. The following generalization produces an interaction matrix that has a linear condition [16], [17]. Starting with the original state-space model (1), a $p$-step ahead state-space model is

$$
\begin{aligned}
x(k+p) & =A^{p} x(k)+\mathbf{C} u_{p}(k) \\
y_{p}(k) & =\mathbf{O} x(k)+\mathbf{T} u_{p}(k)
\end{aligned}
$$

where

$$
u_{p}(k)=\left[\begin{array}{l}
u(k) \\
u(k+1) \\
\vdots \\
u(k+p-1)
\end{array}\right] y_{p}(k)=\left[\begin{array}{l}
y(k) \\
y(k+1) \\
\vdots \\
y(k+p-1)
\end{array}\right]
$$

The matrix $\mathbf{C}$ is an extended controllability matrix, $\mathbf{O}$ an extended observability matrix, and $\mathbf{T}$ a block-Toeplitz matrix of the system Markov parameters. Adding and subtracting the product $\mathbf{M} y_{p}(k)$ to the $p$-step ahead state equation in (15), one obtains

$$
x(k+p)=(\mathbf{C}+\mathbf{M T}) u_{p}(k)-\mathbf{M} y_{p}(k)
$$

if a generalized interaction matrix $\mathbf{M}$ exists such that $A^{p}+\mathbf{M O}=0$. As long as $p$ is sufficiently large and the system is observable so that $\mathbf{O}$ is full rank, the generalized interaction matrix $\mathbf{M}$ is guaranteed to exist. It can be shown that the coefficients of the ARX model are related to the state-space model (1) and the generalized interaction matrix $\mathbf{M}$ according to $\left[\alpha_{p} \cdots \alpha_{2} \alpha_{1}\right]=-C \mathbf{M}$ and $\left[\beta_{p} \cdots \beta_{2} \beta_{1}\right]=C(\mathbf{C}+\mathbf{M T})$. Key advantages of the generalized interaction matrix include easy determination of its existence because $A^{p}+\mathbf{M O}=0$ is a linear relationship, simple generalization to multiple-step ahead prediction for Model Predictive Control (MPC), and straightforward extension to time-varying state-space models.

\section{System Identification in the Presence of Periodic Disturbances}

So far the interaction matrices are used to eliminate the state-dependent term in an input-output equation and to limit the number of unknowns. We now show how it can be used to eliminate unknown periodic disturbances in an input-output relationship between excitation input and disturbance-corrupted output. Consider the problem of identifying the disturbance-free model in the presence of unknown periodic disturbances that appear as additional unknown inputs $d(k)$,

$$
\begin{aligned}
x(k+1) & =A x(k)+B u(k)+B_{d} d(k) \\
y(k) & =C x(k)+D u(k)
\end{aligned}
$$


Suppose $d(k)$ is comprised of a finite number of sinusoids with unknown frequencies, amplitudes, and phases. The counterpart of (15) is

$$
\begin{aligned}
x(k+p) & =A^{p} x(k)+\mathbf{C} u_{p}(k)+\mathbf{C}^{(d)} d_{p}(k) \\
y_{p}(k) & =\mathbf{O} x(k)+\mathbf{T} u_{p}(k)+\mathbf{T}^{(d)} d_{p}(k)
\end{aligned}
$$

where $\mathbf{C}^{(d)}$ is an extended controllability matrix and $\mathbf{T}^{(d)}$ a block-Toeplitz matrix associated with the disturbance input $d(k)$. A generalized interaction matrix $\mathbf{M}$ is introduced by adding and subtracting the product $\mathbf{M} y_{p}(k)$ to the right hand side of the state equation in (18),

$$
\begin{aligned}
x(k+p)=A^{p} x(k)+\mathbf{C} u_{p}(k)+\mathbf{C}^{(d)} d_{p}(k) & \\
& +\mathbf{M} y_{p}(k)-\mathbf{M} y_{p}(k) \\
=\left(A^{p}\right. & +\mathbf{M O}) x(k)+(\mathbf{C}+\mathbf{M T}) u_{p}(k) \\
& +\left[\mathbf{C}^{(d)}+\mathbf{M T} \mathbf{T}^{(d)}\right] d_{p}(k)-\mathbf{M} y_{p}(k)
\end{aligned}
$$

The freedom introduced by $\mathbf{M}$ is used to eliminate both the explicit dependence of $x(k)$ on the right hand side of (19) and the unknown periodic disturbance input $d(k)$ by setting $A^{p}+\mathbf{M O}=0$ and $\left[\mathbf{C}^{(d)}+\mathbf{M T}^{(d)}\right] d_{p}(k)=0$. If $d(k)$ is made of a finite number of sinusoidal inputs of unknown frequencies, amplitudes, and phases, and $p$ is such that $p m \geq n+2 f+1$ where $f$ is the number of distinct disturbance frequencies and the 1 accounts for a constant bias if present, then $\mathbf{M}$ exists. Therefore, a model relating excitation input and disturbance-corrupted output exists,

$$
y(k)=C(\mathbf{C}+\mathbf{M T}) u_{p}(k-p)-C \mathbf{M} y_{p}(k-p)+D u(k)
$$

for $k \geq p$. Equation (20) itself is an ARX model whose disturbance-corrupted coefficients absorb the disturbance information. The interaction matrix explains the relationship between the disturbance-corrupted ARX model coefficients and the state-space model matrices. This ARX model can be identified from disturbancecorrupted input-output data [17]. Again, the system Markov parameters of the disturbance-free model can be recovered from the disturbance-corrupted ARX model coefficients without having to find the interaction matrix $\mathbf{M}$ itself.

\section{Interaction Matrices for a Bilinear Model}

Bilinear systems have seen a recent spark of interests in the aerospace engineering community. Although one might view bilinear systems as merely a special case of nonlinear systems, the importance of bilinear systems is far greater. Whereas some dynamical systems are inherently bilinear, other nonlinear systems can be converted into bilinear form by a process known as Carleman linearization [44]. The latter application of bilinear system is important because it offers an opportunity to present a very large class of nonlinear systems in a common bilinear form, 


$$
\begin{aligned}
x(k+1) & =A x(k)+B u(k)+N x(k) u(k) \\
y(k) & =C x(k)+D u(k)
\end{aligned}
$$

Much of the challenge in state-space system identification arises from the fact that the states are not known. Our approach is to express the bilinear system state in terms of input-output measurements via an Input-Output-to-State Relationship (IOSR) which itself can be viewed as an observer. Propagating Eq. (21) one step forward produces an expression for $x(k+2)$,

$$
\begin{aligned}
x(k+2)= & A x(k+1)+B u(k+1)+N x(k+1) u(k+1) \\
= & A^{2} x(k)+A N x(k) u(k)+N A x(k) u(k+1)+N^{2} x(k) u(k) u(k+1) \\
& +A B u(k)+N B u(k) u(k+1)+B u(k+1)
\end{aligned}
$$

Propagating one more step produces an expression for $x(k+3)$. There is a rapid build-up of terms involving $x(k)$ in the right hand sides of these expressions. In general, the bilinear system state at any future time step $x(k+p)$ depends on the state at the current time step $x(k)$ which is unknown. Yet in (22) it is not possible to eliminate these state-dependent terms because we have no control over the bilinear system matrices $A$ and $N$. In the following a mechanism to alter the dynamics of $A$ and $N$ is achieved through the use of interaction matrices.

Adding and subtracting $M_{1} y(k)$ and $M_{2} y(k) u(k)$ to the original state equation in (21) produces

$$
\begin{aligned}
x(k+1)= & A x(k)+B u(k)+N x(k) u(k)+M_{1} y(k)-M_{1} y(k) \\
& +M_{2} y(k) u(k)-M_{2} y(k) u(k) \\
= & \left(A+M_{1} C\right) x(k)+\left(B+M_{1} D\right) u(k)-M_{1} y(k) \\
& +M_{2} D u^{2}(k)-M_{2} y(k) u(k)+\left(N+M_{2} C\right) x(k) u(k)
\end{aligned}
$$

The interaction matrices $M_{1}$ and $M_{2}$ alter the original system dynamics according to

$$
\bar{A}=A+M_{1} C \quad \bar{N}=N+M_{2} C \quad \bar{B}=\left[\begin{array}{llll}
B+M_{1} D & -M_{1} & M_{2} D & -M_{2}
\end{array}\right]
$$

and $v(k)=\left[\begin{array}{llll}u(k) & y(k) & u^{2}(k) & y(k) u(k)\end{array}\right]^{T}$ so that the original system now is:

$$
\begin{aligned}
x(k+1) & =\bar{A} x(k)+\bar{B} v(k)+\bar{N} x(k) u(k) \\
y(k) & =C x(k)+D u(k)
\end{aligned}
$$

The freedom introduced by the interaction matrices is used to eliminate the statedependent terms. These interaction matrices themselves do not need to be found, but they create expressions that relate the bilinear system state $x(k)$ in terms of a known vector consisting of input-output measurements called a superstate vector $z(k), x(k)=T z(k)$. Other interaction matrices are involved in producing other IOSR's including those are non-causal, or mixed causal and non-causal [35]-[42]. 


\section{Interaction Matrices in Bilinear System Identification}

There are at least five different strategies that these IOSRs can be used to produce a large number of bilinear system identification methods. First, they can convert the bilinear model to an equivalent linear model (ELM) [32]. The known superstate $z(k)$ combined with $u(k)$ in the product $z(k) u(k)$ can be treated as anditional input to a linear model which can be identified by linear system identification techniques,

$$
\begin{aligned}
x(k+1) & =A x(k)+\left[\begin{array}{ll}
B & N T
\end{array}\right]\left[\begin{array}{c}
u(k) \\
z(k) u(k)
\end{array}\right] \\
y(k) & =C x(k)+D u(k)
\end{aligned}
$$

Second, the IOSR's can be used to create a nonlinear ARX (NARX) model [33]

$$
y(k)=C T z(k)+D u(k)
$$

The coefficients of the NARX model contained in $C T$ and $D$ can be identified from input-output data as packaged in $z(k)$ and $u(k)$. From these coefficients, the Markov parameters of the bilinear model can be recovered (without having to find the interaction matrices) from which a bilinear state-space model is found using standard realization theory. Third, certain superstates $z(k)$ 's associated with these IOSRs can be used directly to identify a non-minimum order bilinear state-space model of the system with $z(k)$ treated as a known state variable [34], [38],

$$
\begin{gathered}
z(k+1)=A_{r} z(k)+B_{r} u(k)+N_{r} z(k) u(k) \\
y(k)=C_{r} z(k)+D u(k)
\end{gathered}
$$

The identification of the bilinear state-space model becomes a simple linear problem if the bilinear state is known. Model reduction then follows to reduce identified bilinear model to the minimum dimension. This is a superspace method. Fourth, different representations of the superstates can be intersected to find the bilinear system state from which simple least-squares method can be used to identify a bilinear state-space model [36]-[38]. This is a subspace intersection method which can be explained as follows. Consider two suitable IOSR's, $x(k)=T_{1} z_{1}(k)$ and $x(k)=T_{2} z_{2}(k)$. Let $X$ denote a matrix whose columns are the bilinear system state at different time steps,

$$
X=[x(k) x(k+1) x(k+2) \cdots]
$$

and $Z_{1}$ and $Z_{2}$ are matrices whose columns are the corresponding superstates,

$$
\begin{aligned}
& Z_{1}=\left[z_{1}(k) z_{1}(k+1) z_{1}(k+2) \cdots\right] \\
& Z_{2}=\left[z_{2}(k) z_{2}(k+1) z_{2}(k+2) \cdots\right]
\end{aligned}
$$

If the rows of $X$ reside in the row spaces of $Z_{1}$ and of $Z_{2}$, then they must reside in the intersection of the row spaces of $Z_{1}$ and $Z_{2}$. By finding the intersection subspace 
of $Z_{1}$ and $Z_{2}$, we can find $X$. Fifth, the IOSR's can also be used to produce subspace projection algorithms [39] by rewriting the bilinear state-space model as,

$$
x(k+1)=A T_{1} z_{1}(k)+\left[\begin{array}{ll}
B & N T_{2}
\end{array}\right]\left[\begin{array}{c}
u(k) \\
z_{2}(k) u(k)
\end{array}\right]
$$

With proper choices for the two IOSR's, it is straightforward to derive subspace projection algorithms that find the combination $\Gamma X$, a product of an observability matrix $\Gamma$ and the state history matrix $X$, as an oblique projection of the rows of an output data matrix onto the subspace defined by $z_{1}(k)$ along the subspace defined by $u(k)$ and $z_{2}(k) u(k)$. A factorization of $\Gamma X$ then produces the bilinear system state history $X$. Yet another approach to bilinear system identification views it as a linear system with an input-dependent system matrix. If the input is restricted to a finite set of values, the bilinear system becomes a switched linear systems [37]-[39]. The generalized interaction matrices which are suitable for linear time-varying systems can be used in these switched linear systems to produce IOSR's from which any of the five strategies described previously can be employed. The switched linear system approach produces methods that identify a discrete bilinear system exactly.

\section{Conclusions}

This paper is a brief introduction to the interaction matrices as related to our own work in linear and bilinear system identification. The interaction matrices that appear in various applications share a unifying theme. Certain input-output models or input-output-to-state models are needed for a specific application. The interaction matrices provide a mechanism to determine if such models exist, and how they can be derived from a given state-space model, or identified from input-output measurements. In most applications, the interaction matrices can remain implicit, and only their existence need to be assured. In other applications, they carry specific interpretations such as observer or Kalman filter gains that can be recovered from the identified input-output model coefficients. Input-output or input-output-to-state models are integral to the solution of many problems. The ability of the interaction matrices to tailor these models to specific needs makes them very useful in a wide range of system identification and control applications.

\section{References}

1. Chen, C.W., Huang, J.-K., Phan, M.Q., and Juang, J.-N.: Integrated System Identification and Modal State Estimation for Control of Large Flexible Space Structures. Journal of Guidance, Control, and Dynamics, 15(1), 88-95 (1992)

2. Phan, M.Q., Juang J.-N., and Longman, R.W.: Identification of Linear Multivariable Systems by Identification of Observers with Assigned Real Eigenvalues. Journal of the Astronautical 
Sciences, 40(2), 261-27 (1992)

3. Phan M.Q., Horta, L.G., Juang, J.-N., and Longman, R.W.: Linear System Identification Via An Asymptotically Stable Observer. Journal of Optimization Theory and Applications, 79(1), 59-86 (1993)

4. Juang, J.-N., Phan, M.Q., Horta, L.G., and Longman, R.W.: Identification of Observer/Kalman Filter Markov Parameters: Theory and Experiments. Journal of Guidance, Control, and Dynamics, 16(2), 320-329 (1993)

5. Chen, C.-W., Juang, J.-N., and Phan, M.Q.: Estimation of Kalman Filter Gain from Output Residuals. Journal of Guidance, Control, and Dynamics, 16(5), 903-908 (1993)

6. Phan M.Q., Horta, L.G., Juang, J.-N., and Longman, R.W.: Improvement of Observer/Kalman Filter Identification (OKID) by Residual Whitening. Journal of Vibrations and Acoustics, 117, 232-238 (1995)

7. Lim, R.K., Phan, M.Q., and Longman, R.W.: State Estimation with ARMarkov Models. Department of Mechanical and Aerospace Engineering Technical Report No. 3046, Princeton University (1998)

8. Lim, R.K., Phan, M.Q., and Longman, R.W.: State Space System Identification with Identified Hankel Matrix. Department of Mechanical and Aerospace Engineering Technical Report No. 3045, Princeton University, (1998)

9. Phan, M.Q., Solbeck, J., and Ray, L.: A Direct Method for State-Space Model and Observer/Kalman Filter Gain Identification. Proceedings of the AIAA Guidance, Navigation, and Control Conference and Exhibit, Providence, RI (2004)

10. Vicario, F., Phan, M.Q., Raimondo, B., and Longman, R.W.: OKID as a Unified Approach to System Identification. Advances in the Astronautical Sciences. 152, 3443-3460 (2014)

11. Vicario, F., Phan, M.Q., Raimondo, B. and Longman, R.W.: Output-Only Observer/Kalman Filter Identification $\left(\mathrm{O}^{3} \mathrm{KID}\right)$. Journal of Structural Control and Health Monitoring. Online: DOI 10.1002/stc.1719, (2014)

12. Juang J.-N. and Phan, M.Q.: Identification of System, Observer, and Controller from Closedloop Experimental Data. Journal of Guidance, Control, and Dynamics, 17(1), 91-96 (1994)

13. Phan, M.Q., Juang, J.-N., Horta, L.G., and Longman, R.W.: System Identification from Closed-Loop Data with Known Output Feedback Dynamics. Journal of Guidance, Control, and Dynamics, 17(4), 661-669 (1994)

14. Juang J.-N. and Phan, M.Q.: Linear System Identification Via A Backward-Time Observer. Journal of Guidance, Control, and Dynamics, 17(3), 505-512 (1994)

15. Lim R.K. and Phan, M.Q.: Identification of a Multistep-Ahead Observer and Its Application to Predictive Control. Journal of Guidance, Control, and Dynamics, 20(6), 1200-1206 (1997)

16. Phan M.Q., Lim R.K., and Longman, R.W.: Unifying Input-Output and State-Space Perspectives of Predictive Control. MAE Department Technical Report No. 3044, Princeton Univ., (1998) (http://www.dartmouth.edu/mqphan/predctr.html).

17. Goodzeit N.E. and Phan, M.Q.: System Identification in the Presence of Completely Unknown Periodic Disturbances. Journal of Guidance, Control, and Dynamics, 23(2), 251-259 (2000)

18. Goodzeit N.E. and Phan, M.Q.: System and Disturbance Identification for FeedforwardFeedback Control Applications. Journal of Guidance, Control, and Dynamics, 23(2), 260-268 (2000)

19. Goodzeit N.E. and Phan, M.Q.: An Intelligent Adaptive System for Flexible Spacecraft Identification and Disturbance Rejection. Journal of Vibration and Control, 6, 757-780 (2000)

20. Phan, M.Q., Longman, R.W., Lee, S.C., and Lee, J.W.: System Identification from Multiple Trial Data Corrupted by Non-Repeating Periodic Disturbances. International Journal of Applied Mathematics and Computer Science, 13(2), 185-192 (2003)

21. Koh, B.H., Dharap, P., Nagarajaiah, S., and Phan, M.Q.: Real-Time Structural Damage Monitoring by Input Error Function. AIAA Journal, 43(8), 1808-1814 (2005)

22. Koh, B.H., Li, Z., Dharap, P., Nagarajaiah, S., and Phan, M.Q.: Actuator Failure Detection Through Interaction Matrix Formulation. Journal of Guidance, Control, and Dynamics, 28(5), 895-901 (2005) 
23. Darling R.S. and Phan, M.Q.: Predictive Controllers for Simultaneous Tracking and Disturbance Rejection. Proceedings of the AIAA Guidance, Navigation, and Control Conf. and Exhibit, Providence, RI, (2004)

24. Darling R.S. and Phan, M.Q.: Decentralized Predictive Control for Tracking and Disturbance Rejection. Proceedings of the AIAA Guidance, Navigation, and Control Conf. and Exhibit, Keystone, CO, (2006)

25. Darling R.S. and Phan, M.Q.: Tracking Control of Unmeasured Outputs. Proceedings of the AIAA Guidance, Navigation, and Control Conference and Exhibit, Keystone, CO, (2006)

26. Barlow J.S. and Phan, M.Q.: Adaptive Predictive Control of Vehicle Formations. Advances in the Astronautical Sciences, 129, 2517-2536 (2007)

27. Barlow J.S. and Phan, M.Q.: Model-Based Predictive Control of Vehicle Formations. Advances in the Astronautical Sciences, 129, 2007, 2407-2516 (2007)

28. Phan, M.Q. and Juang, J.-N.: Designs of Learning Controllers Based on an Auto-Regressive Representation of a Linear System. Journal of Guidance, Control, and Dynamics, 19(2), 355362 (1996)

29. Phan, M.Q., Longman, R.W., and Juang, J.-N.: Identification of Linear Time-Varying Systems by Canonical Representation. Advances in the Astronautical Sciences, 135, 1401-1415 (2010)

30. Phan, M.Q., Longman, R.W., and Juang, J.-N.: A Direct Method For Identifying Linear TimeVarying State-Space Models. Advances in the Astronautical Sciences,135, 161-174 (2010)

31. Phan, M.Q., Shi, Y., Betti, R., and Longman, R.W.: Discrete-Time Bilinear Representation of Continuous-Time Bilinear State-Space Models. Advances in the Astronautical Sciences, 143, 571-589 (2012)

32. Hizir, B., Phan, M.Q., Betti, R., and Longman, R.W.: Identification of Discrete-Time Bilinear Systems Through Equivalent Linear Models. Nonlinear Dynamics, 69(4), 2065-2078 (2012)

33. Celik H. and Phan, M.Q.: Identification of Input-Output Maps for Bilinear Discrete-Time State-Space Models. Advances in the Astronautical Sciences, 142, 393-412 (2012)

34. Lin P., Phan, M.Q., and Ketcham, S.A.: State-Space Model and Kalman Filter Gain Identification by a Superspace Method. Modeling, Simulation, and Optimization of Complex Processes, Bock, H.G., Phu, H.X., Rannacher, R., and Schloeder, J., Editors, Springer, 121-132 (2014)

35. Phan, M.Q. and Čelik, H.: A Superspace Method for Discrete-Time Bilinear Model Identification by Interaction Matrices. Journal of the Astronautical Sciences, 59(1,2), 433-452 (2012)

36. Vicario, F., Phan, M.Q., Raimondo, B. and Longman, R.W.: Linear State Representation for Identification of Bilinear Discrete-Time Models by Interaction Matrices. Nonlinear Dynamics, 77(4), 1561-1576 (2014)

37. Vicario, F., Phan, M.Q., Longman, R.W. and Raimondo, B.: A Linear Time-Varying Approach for Exact Identification of Bilinear Discrete-Time Systems by Interaction Matrices. Advances in the Astronautical Sciences, 150,1056-1076 (2014)

38. Phan, M.Q., Vicario, F., Longman, R.W. and Raimondo, B.: Superspace and Subspace Intersection Identification of Bilinear Systems with Discrete-Level Inputs. AAS/AIAA Astrodynamics Specialist Conference, Vail, CO. (2015).

39. Phan, M.Q., Vicario, F., Longman, R.W. and Raimondo, B.: A Subspace Projection Algorithm for Identification of Bilinear Systems with Discrete-Level Inputs. In preparation.

40. Phan, M.Q., Vicario, F., Longman, R.W., and Betti, R.: Optimal Bilinear Observers for Bilinear State-Space Models by Interaction Matrices. International Journal of Control, Online: DOI 10.1080/00207179.2015.1007530. (Print version to appear), (2015)

41. Vicario, F., Phan, M.Q., Longman, R.W. and Betti, R.: Bilinear System Identification by Minimal-Order State Observers. The 25th AAS/AIAA Space Flight Mechanics Meeting, Williamsburg, VA, (2015)

42. Vicario, F., Phan, M.Q., Betti, R. and Longman, R.W.: Bilinear Observer/Kalman Filter Identification. Advances in the Astronautical Sciences, 152, 1517-1536 (2014)

43. Phan, M.Q.: Interaction Matrices in System Identification and Control. Proceedings of the15th Yale Workshop on Adaptive and Learning Systems, New Haven, CT, (2011)

44. Kowalski, K. and Steeb W.-H.: Nonlinear Dynamical Systems and Carleman Linearization, World Scientific (1991) 